\section{EXCITING NEW SEATING OPTIONS}

Meditelle-Dental are introducing exciting new options on their popular dental seating models.

Medi Saddles with back and torso supports will be available with a pneumatic foot height control, offered previously on the standard

Medi Stool. In response to requests from dental surgeons the pneumatic foot control with hands free height adjustment will also be offered on the popular Tri-seating range.

Meditelle-Dental, who bespoke manufacture specialist seating in their Birmingham UK factory, will also offer a leather hide covering option on all saddle seating from April onwards. Reader response number 50
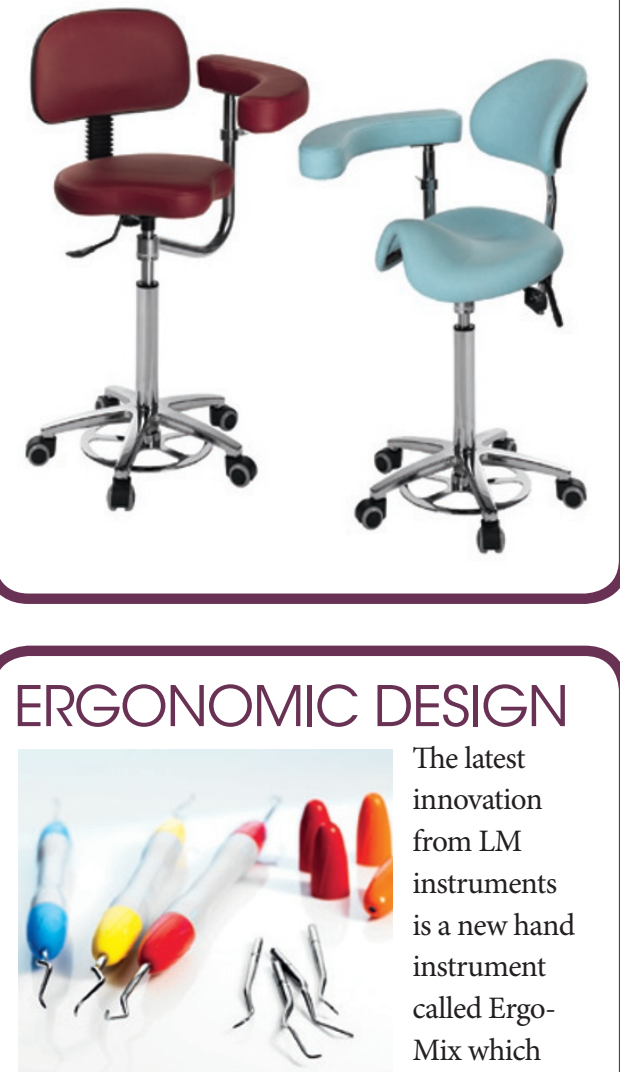

The latest

innovation

from LM

instruments

is a new hand

instrument

called Ergo-

Mix which

offers clinicians

an economical, ecological and ergonomic alternative to standard dental instruments. The simple colour coded lock-grip system allows a quick change of tips without the need for tools. The advanced DuraGrade tips offer long lasting sharpness which coupled with the ergonomic design offers possibly the most advanced hand instrument available today.

In addition LM is supplying the Ergomax range of instruments which are particularly popular with dental hygienists and therapists. Reader response number 53

\section{REPAIR AND PROTECT}

New Sensodyne Repair \& Protect comes from Sensodyne, specialists in dentine hypersensitivity, and is powered by NovaMin. This exciting new variant within the Sensodyne range not only delivers clinically proven relief from

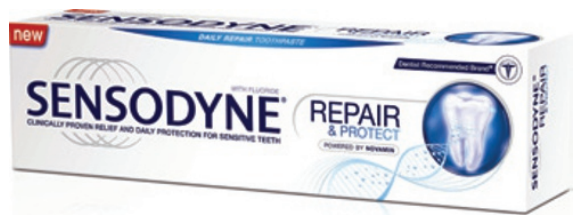
the pain of dentine hypersensitivity but also goes beyond pain treatment to help repair exposed dentine, providing substantive protection against future damage.

Clinical studies have shown that a $5 \%$ Novamin formulation is effective in preventing the pain of sensitive teeth. NovaMin helps build a reparative hydroxyapatite-like layer over exposed dentine and within the tubules. This layer, formed by Sensodyne Repair \& Protect, uses the natural building blocks of teeth and starts to form from the first use. The layer can withstand daily oral challenges such as toothbrushing and acidic food and drinks to help provide continual protection from pain with twice daily brushing. Reader response number 51

\section{SUPERIOR PLAQUE CONTROL}

The unique formula of triclosan and copolymer in Colgate Total provides superior plaque control for a healthier mouth, and continues to meet the evolving needs of dental professionals and their patients.

The new and improved Colgate Total continues to provide superior plaque reduction for better gingival health. Now in addition, it protects sensitive teeth by sealing opening tubules, is more effective at reducing supra-gingival calculus build up, and has improved stain removal properties. Colgate Total contains $1450 \mathrm{ppm}$ sodium fluoride providing maximum caries control and protection against erosion.

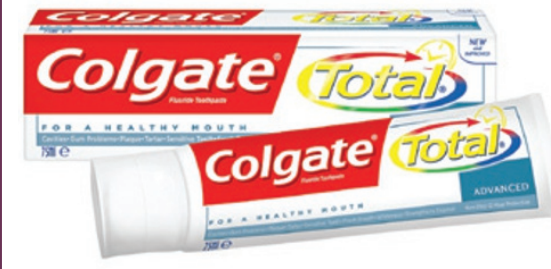

Now you can protect your patients from the most common and preventable dental problems by recommending new and improved Colgate Total toothpaste reducing plaque, calculus, dental caries, gum problems, sensitive teeth, enamel erosion, bad breath and staining. Reader response number 52

\title{
CREDIT CARD SIZED DENTAL FLOSS
}

FlossFirst is 60 metres of high tenacity dental floss enclosed in a credit card shaped container: literally 'word of mouth advertising'.

FlossFirst can be printed with your dental practice's logos, photos, artwork, maps, lists of services, appointment details and the Australian suppliers can also provide artwork for your consideration, obligation free. Educational 'how to' graphics can be colour coded and are complimentary. When the artwork is approved, payment is required, and then the product is printed, packed and posted airfreight to your door.

For the dental profession FlossFirst is a recall aid: hold the card to the light and you will see the amount left. If low, it is time for the patient to make an appointment. This subtle approach helps keep your appointment book filled.

Each credit card shaped container is individually packed in a plastic wallet and is priced at $£ 1.20$ for the UK market.

Reader response number 54

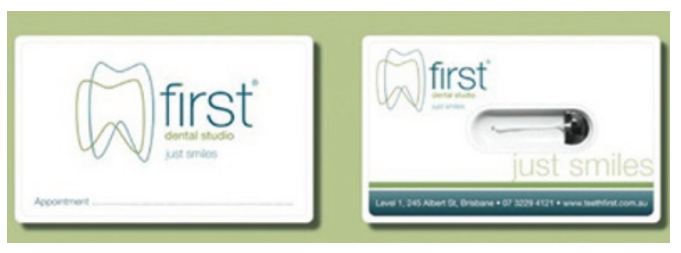

